\title{
HIV-related stigma experienced by people living with HIV/AIDS in coastal South India
}

\author{
Arjun B. Yathiraj ${ }^{1}$, Bhaskaran Unnikrishnan ${ }^{1}$, John T. Ramapuram² ${ }^{2}$, Nithin Kumar ${ }^{1}$, Prasanna Mithra ${ }^{1}$, \\ Rekha Thapar ${ }^{1}$, Vaman Kulkarni ${ }^{1}$, Ramesh Holla ${ }^{1}$, Darshan B.B. ${ }^{1}$, Prasanth Narahari ${ }^{1}$ \\ ${ }^{1}$ Department of Community Medicine, Kasturba Medical College (affiliated to Manipal Academy of Higher Education), Mangalore, \\ Karnataka, India \\ ${ }^{2}$ Department of Internal Medicine, Kasturba Medical College (affiliated to Manipal Academy of Higher Education), Mangalore, \\ Karnataka, India
}

\begin{abstract}
Introduction: The present study assessed the human immunodeficiency virus (HIV)-related stigma and the factors influencing it among people living with human immunodeficiency virus (PLHIV) in tertiary care hospitals at Mangalore, South India. Stigma related to HIV still persists as a major public health issue globally and varies from person to person. The various forms of HIV-related stigma are personalized stigma, disclosure stigma, negative self-image stigma, and public attitude stigma.

Material and methods: In this cross-sectional study, 409 PLHIV aged $\geq 18$ years were interviewed from April 2014 to April 2015. HIV-related stigma was assessed using the "revised HIV stigma scale". Socio-demographic characteristics and factors affecting HIV stigma were collected using a semi-structured questionnaire. The association between various domains of HIV-related stigma with socio-demographic characteristics was assessed using one way ANOVA (analysis of variance) and student $t$-test. $P<0.05$ was considered to be statistically significant.

Results: Among the HIV-related stigma domains, disclosure and public attitudes stigma showed higher mean scores followed by negative self-image stigma. Personalized stigma domain showed the least scores in our study. Our study identified that HIV-related stigma was higher among males, older participants, those who were married, of a higher socio-economic status, on a longer duration of antiretroviral therapy (ART), and participants who had travelled more distance to procure ART.

Conclussions: Counseling PLHIV and educating care givers and the community is essential for tackling stigmatization among PLHIV. Mitigating stigma through multi-dimensional intervention strategies is crucial to overcome stigma associated with HIV/AIDS.
\end{abstract}

HIV AIDS Rev 2018; 17, 1: 30-35

DOI: https://doi.org/10.5114/hivar.2018.73359

Key words: HIV-related stigma, revised HIV stigma scale, PLHIV, South India.

\section{Introduction}

Human immunodeficiency virus/acquired immunodeficiency syndrome (HIV/AIDS) is a major public health prob-

Address for correspondence: Prof. Bhaskaran Unnikrishnan, Department of Community Medicine, Kasturba Medical College (affiliated to Manipal Academy of Higher Education), Light House Hill Road, Hampankatta, 575001 Mangalore (Karnataka), India, phone: 919880530700, e-mail: unnikrishnan.b@manipal.edu

lem affecting as many as 36.7 million people worldwide [1]. According to National AIDS Control Organization (NACO), India has around 2.1 million HIV-infected individuals, with HIV adult prevalence of $0.26 \%$ at the end of 2015 [2].

Article history:

Received: 22.09.2017

Received in revised form: 02.11.2017

Accepted: 03.11.2017

Available online: 30.01 .2018
International Journal of HIV-Related Problems

HIV \& AIDS

$R$ e $v$ i e w 
HIV-related stigma among people living with human immunodeficiency virus (PLHIV) has been a complicated issue globally since the beginning of its discovery.

In recent times, from a fatal illness, HIV/AIDS has been reduced to a chronic, manageable disease with the availability of effective antiretroviral therapy (ART). However, stigma related to the disease still continues to persist amongst all sections of the society. PLHIV are more stigmatized compared to those with other chronic diseases [3], as HIV is a communicable disease associated with sexual and other high-risk behavior and lacks a definite cure.

PLHIV not only face problems related to physical health, but also experience many psychological issues like depression, anxiety, fear, anger, and feelings of isolation [4]. HIV-related stigma interferes with the health seeking behavior of PLHIV, leading to poor adherence to ART, decreasing quality of life and in turn, increasing morbidity and mortality [5].

HIV-related stigma experienced by PLHIV can be categorized mainly into four domains of stigma. Personalized stigma, where PLHIV experiences fear about the consequences of what others might think about their HIV status and societal attitudes about their HIV status.

Disclosure stigma, where PLHIV are concern about disclosing their HIV status, keeps one's HIV status secret, or fear that those who know about their HIV status will tell others. HIV status disclosure is one of the major psychological challenges for PLHIV [6]. Disclosure stigma also introduces a desire not to get HIV-tested to know one's own sero-status. Thus, it possibly increases the risk of infection to their sexual partners, and also PLHIV becomes not avail to HIV care and treatment services due to disclosure stigma.

Negative self-image refers to feeling of not as good as others, feeling unclean or as bad person due to being HIVinfected. PLHIV may also experience guilt, shame, and selfworth due to negative self-image stigma.

Public attitude stigma is a form of stigma where PLHIV are concerned about what most people might think about their HIV status, or what most people think about HIV-infected person.

Ultimately, whatever maybe the form of stigma, it leads to poor adherence to ART, decreased quality of life, which may lead to increased morbidity and mortality [4].

The presence of stigma among PLHIV has been documented by many studies [7-9]. However, stigma experienced by PLHIV greatly varies considerably among the individuals and their surroundings. Proper knowledge about how HIV-related stigma perpetuates is essential for planning culturally and socially relevant intervention strategies in a particular geographical area. The present study was conducted to assess the factors associated with HIV/AIDS-related stigma experienced by PLHIV in coastal South India.

\section{Material and methods}

In this facility-based cross-sectional study, PLHIV attending Infectious Disease Department of the Kasturba Medical
College Hospital (KMCH), Mangalore, Karnataka were interviewed during the period from April 2014 to April 2015, to assess their HIV-related stigma. The infectious disease department of $\mathrm{KMCH}$ is one of the nodal center for providing counseling and ART services to PLHIV.

The protocol of the study was approved by the Institutional Ethics Committee (IEC) at Kasturba Medical College (Manipal Academy of Higher Education), Mangalore, India, prior to the commencement of the study.

The sample size of 409 PLHIV was calculated considering the proportion of HIV-related stigma among PLHIV from the previous study as $27 \%$ [10], with $95 \%$ confidence interval and a relative precision of $15 \%$. The participants were selected using non-random sampling method.

PLHIV in the age group of 18 years and above, and those who are receiving ART were included in the study. No in-patients were included in the study.

The participants were explained about the study objectives in their own vernacular language and written informed consent was obtained from those who were willing to participate in the study. The interview was conducted in a separate consultation room to maintain patient's confidentiality. The average duration of the interview was 30 minutes.

Interview schedule consisted of questions pertaining to socio-demographic characteristics (age, gender, marital status, socioeconomic status, duration on ART, and distance travelled to receive ART) and HIV-related stigma. Modified Kuppuswamy scale [11] was used to assess socio-economic status of PLHIV, which includes education, occupation, and income of the participants.

\section{HIV-related stigma}

HIV-related stigma among PLHIV was assessed using the revised HIV stigma scale [12], which consisted of 10-items. The scale assessed HIV-related stigma across 4 domains as follows: personalized stigma (3-items), disclosure concerns (2-items), negative self-image (3-items), and public attitudes (2-items). The scores for each item of revised HIV stigma scale was analyzed using four-point Likert scale, where: 1 = strongly disagree, 2 = disagree, 3 = agree, and 4 = strongly agree. The mean scores for each domain were obtained by adding the response of each item and dividing it by the number of items. Higher scores indicate greater stigma experienced by the PLHIV.

\section{Data analysis}

Statistical analysis was done using Statistical Package for Social Sciences (SPSS) version 16.0 (SPSS. Inc., Chicago. IL, USA). The results were expressed in mean, standard deviation (SD), and proportion. The association of various domains of HIV-related stigma with socio-demographic characteristics was assessed using one way ANOVA and student $t$-test. $P<0.05$ was considered to be statistically significant. 


\section{Results}

Among 409 PLHIV, the majority $(62.6 \%, n=256)$ being males. The mean age of the participant was $43 \pm 8.7$, with higher proportion of the participants $(42.1 \%, n=172)$ were in the age group of $41-50$ years, and $64.8 \%$ were married.

\section{Descriptive statistics of all the items of revised HIV stigma scale}

The mean (SD) of all the items of the revised HIV stigma scale are shown in Table 1.

\section{HIV-related stigma domains scores of the participants}

The mean (SD) of the HIV stigma domains namely personalized stigma, disclosure stigma, negative self-image, and public attitudes stigma are shown in 0 (Table 2).

\section{Association between HIV-related stigma domains with patient's socio-demographic characteristics}

Males had shown higher mean scores in all the stigma domains compared to females, and a statistically significant association $(p<0.05)$ was observed in the domains of personalized stigma and negative self-image. Participants who were married had higher domain scores compared to those who were unmarried and widowed, and also participants with upper socio-economic status had higher scores in all the domains of HIV-related stigma compared to those with lower socio-economic status.

A statistically significant association $(p<0.05)$ was observed with marital status and socio-economic status of the participants in disclosure stigma and negative self-image stigma domains. PLHIV who were receiving ART for longer duration had higher scores in all the domains of HIV-related stigma with statistically significant association in personalized stigma, negative self-image, and public attitudes stigma domains. Among PLHIV who were travelled more distance $>25$ kilometers $(\mathrm{kms})$ to receive ART, higher HIV stigma domain scores were observed. Association between HIV-related stigma domains with patient's characteristics of the participants is shown in Table 3.

\section{Discussion}

In the present study, PLHIV experienced mainly disclosure and public attitudes stigma, followed by negative self-image stigma. They faced less personalized stigma

Table 1. Descriptive statistics for all the items of revised HIV stigma scale $(n=409)$

\begin{tabular}{|c|c|c|}
\hline No. of items & Items of revised HIV stigma scale & Mean (SD) \\
\hline \multicolumn{3}{|c|}{ Personalized stigma } \\
\hline 01 & I have been hurt by how people reacted to learning I have HIV & $2.6(0.7)$ \\
\hline 02 & I have stopped socializing with some people because of their reactions to my having HIV & $2.3(0.8)$ \\
\hline 03 & I have lost friends by telling them I have HIV & $2.0(0.7)$ \\
\hline \multicolumn{3}{|c|}{ Disclosure stigma } \\
\hline 04 & I am very careful who I tell that I have HIV & $3.6(0.5)$ \\
\hline 05 & I worry that people who know I have HIV will tell others & $3.6(0.5)$ \\
\hline \multicolumn{3}{|c|}{ Negative self-image } \\
\hline 06 & I feel I am not as good a person as others because I have HIV & $2.7(0.7)$ \\
\hline 07 & Having HIV makes me feel unclean & $2.7(0.6)$ \\
\hline 08 & Having HIV makes me feel that I'm a bad person & $2.7(0.7)$ \\
\hline \multicolumn{3}{|c|}{ Public attitudes } \\
\hline 09 & Most people think that a person with HIV is disgusting & $3.6(0.5)$ \\
\hline 10 & Most people with HIV are rejected when others find out & $3.6(0.5)$ \\
\hline
\end{tabular}

Table 2. HIV-related stigma domains score $(n=409)$

\begin{tabular}{l|c|c|c}
\hline Stigma domains & No. of items & Possible range & Mean (SD) \\
\hline Personalized stigma & 03 & $4-12$ & $6.9(1.9)$ \\
Disclosure stigma & 02 & $2-8$ & $7.3(1.0)$ \\
Negative self-image & 03 & $4-12$ & $7.1(1.8)$ \\
Public attitudes & 02 & $2-8$ & $7.3(1.1)$ \\
\hline Total score & 10 & $10-40$ & $29.6(5.8)$ \\
\hline
\end{tabular}


Table 3. Association between the domains of stigma with socio-demographic characteristics $(n=409)$

\begin{tabular}{|c|c|c|c|c|c|}
\hline \multirow[b]{2}{*}{ Characteristics } & \multirow[b]{2}{*}{ Number (\%) } & \multicolumn{4}{|c|}{ HIV-related stigma domain scores } \\
\hline & & $\begin{array}{l}\text { Personalized stigma } \\
\text { Mean }( \pm \text { SD) }\end{array}$ & $\begin{array}{l}\text { Disclosure stigma } \\
\text { Mean }( \pm \text { SD) }\end{array}$ & $\begin{array}{l}\text { Negative self-image } \\
\text { Mean }( \pm \mathrm{SD})\end{array}$ & $\begin{array}{c}\text { Public attitudes } \\
\text { Mean }( \pm \text { SD) }\end{array}$ \\
\hline \multicolumn{6}{|l|}{ Gender } \\
\hline Male & $256(62.6)$ & $7.3(1.7)$ & $7.3(1.0)$ & $8.6(1.6)$ & $7.3(1.1)$ \\
\hline Female & $153(37.4)$ & $6.3(2.2)$ & $7.2(1.0)$ & $7.3(2.0)$ & $7.3(1.0)$ \\
\hline$p$ value* & & $<0.001$ & 0.073 & 0.001 & 0.934 \\
\hline \multicolumn{6}{|l|}{ Age (years) } \\
\hline $18-30$ & $024(05.9)$ & $5.7(2.5)$ & $7.2(1.0)$ & $6.9(2.3)$ & $7.3(1.0)$ \\
\hline $31-40$ & $140(34.2)$ & $6.9(1.9)$ & $7.4(1.0)$ & $8.1(2.0)$ & $7.3(1.0)$ \\
\hline $41-50$ & $172(42.1)$ & $6.8(1.8)$ & $7.3(1.0)$ & $8.2(1.8)$ & $7.3(1.1)$ \\
\hline$>50$ & $073(17.8)$ & $7.6(1.8)$ & $7.0(1.0)$ & $8.3(1.3)$ & $7.1(1.1)$ \\
\hline$p$ value ${ }^{\star *}$ & & $<0.001$ & 0.120 & 0.014 & 0.137 \\
\hline \multicolumn{6}{|l|}{ Marital status } \\
\hline Married & $265(64.8)$ & $7.0(1.9)$ & $7.3(1.0)$ & $8.3(1.7)$ & $7.3(1.1)$ \\
\hline Unmarried & $054(13.2)$ & $6.9(2.0)$ & $7.3(1.0)$ & $8.3(2.5)$ & $7.2(1.2)$ \\
\hline Widowed & $090(22.0)$ & $6.7(2.0)$ & $7.1(1.0)$ & $7.5(1.6)$ & $7.2(1.0)$ \\
\hline$p$ value ${ }^{* *}$ & & 0.197 & 0.027 & 0.002 & 0.258 \\
\hline \multicolumn{6}{|l|}{ Socioeconomic status } \\
\hline Upper middle & $042(10.3)$ & $6.8(2.0)$ & $7.6(0.8)$ & $8.6(1.4)$ & $7.6(0.8)$ \\
\hline Middle/lower middle & $203(49.6)$ & $6.9(1.8)$ & $7.3(1.0)$ & $8.3(1.8)$ & $7.3(1.1)$ \\
\hline Lower/upper lower & $159(38.9)$ & $7.1(2.2)$ & $7.2(1.0)$ & $7.8(1.8)$ & $7.2(1.2)$ \\
\hline Lower & $005(01.2)$ & $4.2(1.1)$ & $6.0(0)$ & $4.2(1.6)$ & $6.4(0.9)$ \\
\hline$p$ value ${ }^{\star *}$ & & 0.780 & 0.002 & $<0.001$ & 0.054 \\
\hline \multicolumn{6}{|l|}{ Duration on ART (years) } \\
\hline$<1$ & $092(22.5)$ & $6.6(1.9)$ & $7.1(1.0)$ & $7.7(1.7)$ & $7.2(1.0)$ \\
\hline $2-3$ & $142(34.7)$ & $6.8(2.1)$ & $7.3(1.0)$ & $7.9(1.7)$ & $7.0(1.2)$ \\
\hline$>3$ & $175(42.8)$ & $7.2(1.8)$ & $7.3(1.0)$ & $8.4(1.9)$ & $7.5(1.0)$ \\
\hline$p$ value ${ }^{* *}$ & & 0.007 & 0.080 & 0.001 & 0.007 \\
\hline \multicolumn{6}{|c|}{ Distance travelled to receive ART $(\mathrm{km})$} \\
\hline$>25 \mathrm{~km}$ & $077(18.8)$ & $7.2(1.9)$ & $7.3(1.0)$ & $8.4(1.8)$ & $7.2(1.3)$ \\
\hline$<25 \mathrm{~km}$ & $332(81.2)$ & $6.8(1.9)$ & $7.3(1.0)$ & $8.0(1.9)$ & $7.3(1.0)$ \\
\hline$p$ value $^{*}$ & & 0.168 & 0.487 & 0.139 & 0.813 \\
\hline
\end{tabular}

*Student $t$-test, ${ }^{* *}$ ANOVA test

$A R T$ - antiretroviral therapy

among the HIV-related stigma domains. Similarly, in a study conducted in Coimbatore District of Tamil Nadu, among the women living with HIV, disclosure stigma was found to be high [13]. In contrast, a study done in Nigeria showed more personalized and public attitudes stigma than disclosure and negative self-image stigma [14].

In our study, HIV-related stigma scores were higher among males as opposed to females, with a statistically significant association $(p<0.05)$ amongst personalized and negative self-image stigma. Similar findings were observed in other studies as well $[15,16]$. This could be attributed to the fact that men have a wider social and professional circle, and they might be apprehensive that people will suspect their HIV status because of the physical changes accompanying/brought on by the disease, or side effects of ART. Taking medications regularly at their workplace also might arouse curiosity among their co-workers, as to what ailment they are suffering from. This might lead to stigma, which may not be the case among women who are generally confined to their homes (in rural setups mainly). The guilt of high-risk behavior in the past, which becomes the reason for not only acquiring HIV/AIDS but also transmitting it to their spouses, could be another reason for stigmatization. However, some study results also indicate that females were more stigmatized than males $[17,18]$. 
Personalized and negative self-image stigma increased with the participants' age, and this association was found to be statistically significant. Similar findings were observed in other studies $[15,18]$. This can be attributed to the fact that with an increase in age along with the changes related to aging, there is a reduction in immunity. With a reduction in immunity, the HIV manifestations increase, leading to increased disease expression.

PLHIV who were married have shown higher HIV stig$\mathrm{ma}$, as compared to those who were unmarried or widowed. Due to increased social obligation among married participants, they are more likely to come in contact with more people, which might be a reason for increased stigmatization. On the contrary, unmarried or widowed PLHIV residing with their parents, children, or relatives, have a socially protected environment, and hence have lesser stigmatization.

In most studies, people belonging to upper socio-economic status showed less HIV-related stigma [19, 20], whereas in stark contrast, our study reveals that participants in the upper socio-economic status had higher HIV-related stigma scores compared to participants in the middle/lower socio-economic status. This could be due to the fact that people who are financially stable have an option of discontinuing work. On the other hand, people belonging to middle and lower socio-economic status don't have a choice but to continue work and provide support for their families. These activities keep them motivated and less stigmatized, rather than brooding over their past or about their future problems in relation to their HIV status. PLHIV with middle/lower socio-economic status experiencing fewer stigmas, could be due to their involvement as a member of social support groups. These groups could have helped them in getting more information about HIV and its treatment, and developing better coping mechanisms to overcome the stigma.

It was observed that PLHIV who travelled long distances (more than 25 kilometers) to receive ART felt more stigmatized compared to those who have travelled less distances. The reason for this could be the fear of revealing their HIV status to their families, friends, or relatives. As a result, they opted for treatment at centers far away from their residing places.

It was also observed that PLHIV receiving ART for a longer duration ( $>3$ years) have experienced higher HIV-related stigma compared to PLHIV on a shorter duration on ART. Similar findings were observed in other studies $[15,18]$. The reason for this could be that PLHIV receiving ART for longer duration might feel stigmatized because other people might notice him/her taking ART, which makes them evident that he/she has HIV.

\section{Conclusions}

HIV-related stigma was higher among males, older age, participants who were married, those belonging to a higher socioeconomic status, on longer duration of ART, and those who had travelled longer distances to obtain ART. Our study emphasizes that HIV-related stigma needs to be tackled at the earliest, as it might affect the mental health and quality of life of PLHIV, along with their physical wellbeing. Once the reasons for stigma have been identified, it becomes easier to target the population and mitigating the stigma associated with this disease. Counseling PLHIV and educating care givers and the community is essential for tackling stigmatization among PLHIV. Mitigating HIV-related stigma through multi-dimensional intervention strategies involving the doctors, health care workers, researchers, social workers, counselors, and nurses, is crucial to overcome stigma associated with HIV/AIDS.

\section{Acknowledgements}

The authors are grateful to the study participants who voluntarily took part in the study. We wish to acknowledge the support provided by the Department of Community Medicine, Kasturba Medical College, Mangalore and Manipal Academy of Higher Education for encouraging research, and its publication in National and International journals.

\section{Conflict of interest}

The authors declare no potential conflicts of interest with respect to the research, authorship, and/or publication of this article.

\section{References}

1. UNAIDS (United Nations Programme on HIV and AIDS). Global Fact Sheet, 2015. Retrieved from: http://www.unaids.org/sites/ default/files/media_asset/UNAIDS_FactSheet_en.pdf.

2. NACO (National AIDS Control Organization). India HIV Estimations 2015. Retrieved from: http://www.naco.gov.in/upload/2015\%20 MSLNS/HSS/India\%20HIV\%20Estimations\%202015.pdf.

3. Mahendra VS, Gilborn L, Bharat S, et al. Understanding and measuring AIDS-related stigma in health care settings: a developing country perspective. Sahara J 2007; 4: 616-625.

4. Kumar S, Mohanraj R, Rao D, et al. Positive coping strategies and HIV-related stigma in South India. AIDS Patient Care STDS 2015; 29: 157-163.

5. Ware NC, Wyatt MA, Tugenberg T. Social relationships, stigma and adherence to antiretroviral therapy for HIV/AIDS. AIDS Care 2006; 18: 904-910.

6. Madi D, Gupta P, Achappa B, et al. HIV Status Disclosure among People Living with HIV in the Era of Combination Antiretroviral Therapy (cART). J Clin Diagn Res 2015; 9: 14-16.

7. Lichtenstein B. Stigma as a barrier to treatment of sexually transmitted infection in the American deep south: issues of race, gender and poverty. Soc Sci Med 2003; 57: 2435-2445.

8. Stevelink S, Wu I, Voorend C, et al. The psychometric assessment of internalized stigma instruments: a systematic review. Stigma Research and Action 2012; 2: 100-118.

9. Mahajan AP, Sayles JN, Patel VA, et al. Stigma in the HIV/AIDS epidemic: a review of the literature and recommendations for the way forward. AIDS 2008; 22: 67-79.

10. Charles B, Jeyaseelan L, Pandian AK, et al. Association between stigma, depression and quality of life of people living with HIV/ AIDS (PLHA) in South India - a community based cross sectional study. BMC Public Health 2012; 12: 463.

11. Dudala SR. Updated Kuppuswamy's socioeconomic scale for 2012. J Dr NTR Univ Health Sci 2013; 2: 201-202. 
12. Wright K, Naar-King S, Lam P, et al. Stigma scale revised: reliability and validity of a brief measure of stigma for HIV+ youth. J Adolesc Health 2007; 40: 96-98.

13. Chitra L, Jayalakshmi L, Vinod R. Stigma in women living with HIV in Coimbatore District of Tamil Nadu. IOSR Journal of Dental and Medical Sciences (IOSR-JDMS) 2015; 13: 29-32.

14. Nworuh OB, Anthony IO. Experience of HIV-related stigma by people living with HIV/AIDS (PLWHA), based on gender: A case of PLWHA attending clinic in the Federal Medical Center, Owerri, Imo states, Nigeria. J Public Health Epidemiol 2013; 5: 435-439.

15. Hasan MT, Nath SR, Khan NS, et al. Internalized HIV/AIDS-related stigma in a sample of HIV-positive people in Bangladesh. J Health Popul Nutr 2012; 30: 22-30.

16. Yebei VN, Fortenberry JD, Ayuku DO. Felt stigma among people living with HIV/AIDS in rural and urban Kenya. Afr Health Sci 2008; 8: 97-102.

17. Swendeman D, Rotheram-Borus MJ, Comulada S. Predictors of HIV-related stigma among young people living with HIV. Health Psychol 2006; 25: 501-509.

18. Nattabi B, Li J, Thompson SC, et al. Factors associated with perceived stigma among people living with HIV/AIDS in post-conflict northern Uganda. AIDS Educ Prev 2011; 23: 193-205.

19. Amuri M, Mitchell S, Cockcroft A, et al. Socio-economic status and HIV/AIDS stigma in Tanzania. AIDS Care 2011; 23: 378-382.

20. Tzemis D, Forrest JI, Puskas CM, et al. Identifying self-perceived HIV-related stigma in a population accessing antiretroviral therapy. AIDS Care 2013; 25: 95-102. 